ORIGINAL

\title{
Conformação dos membros pélvicos de fêmeas bovinas Holandesas em diferentes idades
}

\author{
Conformation of hind legs in female Holstein dairy cattle in different ages
}

\author{
Fernando Passon Casagrande ${ }^{1 *}$, Paulo Marcos Ferreira², Antônio Último de Carvalho², Elias Jorge Facury Filho², \\ Jader Bastos Amorim², Pricilla Pozzatti ${ }^{3}$, Neliton Flores Kasper ${ }^{1}$ \\ ${ }^{1}$ Universidade Federal do Pampa (UNIPAMPA), Uruguaiana, RS, Brasil \\ 2 Universidade Federal de Minas Gerais (UFMG), Belo Horizonte, MG, Brasil \\ ${ }^{3}$ Instituto Federal de Educação, Ciência e Tecnologia Catarinense (IFC), Araquari, SC, Brasil
}

\section{Resumo}

O objetivo deste trabalho foi avaliar a conformação dos membros pélvicos de fêmeas bovinas da raça Holandesa desde o nascimento até a vida adulta. Foram utilizados 210 animais, divididos em sete categorias com 30 animais cada: (C1) bezerras do nascimento até os 3 meses de idade; (C2) bezerras dos 3 aos 6 meses de idade; (C3) bezerras dos 6 meses a 1 ano de idade; (C4) novilhas de 1 ano de idade até o parto; (C5) vacas de uma lactação; (C6) vacas de duas lactações; (C7) vacas de três ou mais lactações. A conformação dos membros pélvicos foi avaliada na sua vista lateral, vista caudal e grau de abdução (locomoção). As conformações dos membros pélvicos na vista caudal e locomoção apresentaram desvios da conformação normal em uma alta proporção nos animais com idade superior a 1 ano, enquanto a conformação dos membros pélvicos na vista lateral apresentou um baixo número de animais com alterações. Conclui-se que a conformação dos membros pélvicos, vista caudal e locomoção apresentou maior variação com a idade e mudança de categoria produtiva dos animais avaliados.

Palavras-chave: Aprumos. Claudicação. Categoria de produção. Faixa etária.

\begin{abstract}
The aim of this work was to evaluate the leg conformation of Holstein dairy cattle from birth to productive life. Therefore, we evaluated 210 female Holstein dairy cattle that were randomly distributed into seven groups (30 per group) according to age and number of lactations: (C1) newborn - 3 months old; (C2) 3 - 6 months old calfes; (C3) 6 months - 1 year old heifers; (C4) 1 year old - to first parturition heifers; (C5) cows with one lactation; (C6) cows with two lactations; (C7) cows with three or more lactations. The leg
\end{abstract}


traits evaluated were rear leg side view, rear leg rear view, and locomotion (abduction) of hind claws. Rear leg, rear view and locomotion of hind claws presented alterations in a large proportion of animals older than one yearof age, while rear leg side view presented alterations in a low proportion of animals. Hence, rear leg, rear view and locomotion presented a higher variation considering age influence and productive category.

Keywords: Leg conformation. Lameness. Production category. Age range.

\section{Introdução}

Claudicação, mastite e distúrbios reprodutivos são os problemas mais onerosos na bovinocultura leiteira (Politiek et al., 1986). Animais com deficiente locomoção não tem fácil acesso à alimentação, diminuindo sua rentabilidade e tendo seu bem-estar afetado (Logue et al., 1993; Warnick et al., 2001). Correlações positivas entre a conformação dos cascos e dos membros pélvicos com a vida do rebanho foram mostradas em vários estudos (Boettcher et al., 1997; Pasman e Reinhardt, 1999). Boettcher et al. (1998) sugerem que a seleção para conformação dos membros pélvicos na vista caudal pode ajudar a reduzir a ocorrência de problemas de locomoção, uma vez que existe alta correlação genética desta característica com a claudicação clínica.

Quando observada a conformação dos membros pélvicos, vacas com cascos saudáveis apresentaram os membros pélvicos mais paralelos e melhor conformação destes na vista lateral do que animais com patologias nestes membros (Olechnowicz et al., 2010). Chapinal et al. (2013), por outro lado, estimaram correlações genéticas de baixas a moderadas entre lesões podais e características de conformação, variando de $-0,39$ para a conformação dos membros pélvicos na vista caudal a 0,26 na vista lateral. Pérez-Cabal e Charfeddine (2016) também observaram que as vacas com escores intermediários de conformação de membros e cascos tiveram uma menor incidência de distúrbios locomotores. Swalve et al. (2008) e Dhakal et al. (2015) encontraram relações genéticas fracas entre a saúde dos cascos e as características da conformação.
A conformação dos membros pélvicos na visão lateral, em particular com a articulação do tarso excessivamente reta, é frequentemente citada como importante fator predisponente na etiologia de lesões nas articulações, tendões e cascos (Greenough et al., 1981). A característica locomoção (grau de abdução dos membros pélvicos) foi adicionada no ano de 2008 às utilizadas nos programas de melhoramento genético da raça Holandesa (WHFF, 2008) e consiste em observar o grau de abdução do dígito dos membros pélvicos, sendo que os animais que apresentam a orientação do espaço interdigital paralela à coluna vertebral têm menor probabilidade de apresentarem problemas. Locomoção e conformação dos membros pélvicos na vista lateral apresentaram altas correlações genéticas com a saúde dos cascos (Laursen et al., 2009).

Na literatura há poucos estudos quantificando os problemas de aprumos dos membros pélvicos, principalmente em animais jovens antes de entrarem na vida produtiva. 0 objetivo deste trabalho, portanto, foi avaliar a conformação dos membros pélvicos de fêmeas bovinas Holandesas desde o nascimento até a vida produtiva, e identificar as mudanças que aparecem com o avanço da idade e do número de lactações.

\section{Materiais e métodos}

\section{Local e condições da fazenda}

O experimento foi desenvolvido em uma fazenda leiteira de criação semi-intensiva, localizada no município de Sete Lagoas, em Minas Gerais. O experimento foi realizado durtante o mês de julho de 2009. Nesse período não houve ocorrência de chuvas que viessem a alterar o ambiente em que os animais estavam. A temperatura mínima nos dias do experimento foi de $9{ }^{\circ} \mathrm{C}$ e a máxima de $32^{\circ} \mathrm{C}$.

\section{Animais utilizados e manejo do rebanho}

Foram avaliadas 210 fêmeas bovinas Holandesas, divididas em sete categorias etárias com 30 animais cada, desde o nascimento até a vida produtiva. Os grupos avaliados foram: (C1) animais 
com menos de 3 meses; (C2) animais com idade entre 3 e 6 meses; (C3) animais com idade entre 6 e 12 meses; (C4) animais entre os 12 meses de idade e o parto; (C5) vacas com uma lactação; (C6) vacas com duas lactações; (C7) vacas com três ou mais lactações.

As bezerras eram criadas em sistema de casinhas, onde permaneciam até os 3 meses de idade, recebendo 2 litros de leite duas vezes ao dia, concentrado e feno à vontade. Após esse período, eram transferidas para piquetes onde eram agrupadas de acordo com a faixa etária e peso até atingirem 8 meses de idade. A alimentação incluía silagem de milho, concentrado e feno. Após os 8 meses de idade, os animais eram transferidos para piquetes de novilhas. Os lotes desta categoria eram compostos de um número maior de animais. Havia nesses piquetes considerável acúmulo de matéria orgânica e umidade ao redor dos comedouros. Os animais permaneciam nesses lotes até um mês antes do período previsto para o parto, quando eram transferidos para os lotes pré-parto de novilhas. Após o parto, os animais eram transferidos para um pequeno free-stall (lote pós-parto), que ficava mais próximo das instalações de ordenha, a fim de facilitar a observação caso ocorresse alguma complicação.

A ordenha era realizada três vezes ao dia. Os animais mais produtivos e os recém-paridos permaneciam em dois pequenos free-stalls perto da sala de ordenha, enquanto o restante permanecia em piquetes com escassa cobertura vegetal; toda a alimentação era fornecida no cocho. Nos piquetes de animais em lactação havia grande acúmulo de matéria orgânica e pouca sombra disponível, providenciada por sombrites. A estrada entre os piquetes dos animais em produção e a sala de ordenha apresentava uma distância aproximada de $500 \mathrm{~m}$, mas como os animais faziam esse percurso de ida e volta três vezes ao dia, caminhavam um total de $3 \mathrm{~km}$ ao dia. 0 caminho dos animais até a sala de ordenha apresentava grande quantidade de pedregulhos. Quando estavam a dois meses do parto, os animais em lactação eram transferidos para os lotes de vacas secas. Quando estavam a um mês do parto, eram transferidos para os lotes préparto de vacas, e após o parto, para o pequeno freestall de animais pós-parto.

\section{Avaliação da conformação dos membros pélvicos}

Atribuiu-se escores de 1 a 3 para conformação dos membros pélvicos na vista lateral, sendo: 1 significa membros pélvicos com ângulo da articulação do tarso maior do que o normal (membros pélvicos muito retos); o escore 2, membros pélvicos com ângulo da articulação do tarso normal; e o escore 3 , membros pélvicos com ângulo da articulação do tarso menor que o normal (membros pélvicos muito curvos). Para a avaliação dos membros pélvicos na vista caudal foram atribuídos escores de 0 a 3, sendo que os membros pélvicos paralelos recebiam escore zero e à medida que as articulações do tarso se aproximavam eram atribuídos escores de 1 a 3 de acordo com a severidade dessa aproximação. Para a avaliação da locomoção (grau de abdução) também foram atribuídos escores de 0 a 3, sendo que os animais que não apresentavam nenhum grau de abdução dos membros recebiam escore 0 e à medida que a abdução aumentava, os escores aumentavam até o limite de 3 pontos. A distribuição dos escores de conformação dos membros pélvicos está apresentada com maiores detalhes na Tabela 1.

Tabela 1 - Distribuição dos escores de conformação dos membros pélvicos, de acordo com a severidade da alteração dos animais de uma fazenda leiteira semi-intensiva com rebanho Holandês do município de Sete Lagoas, MG (julho de 2009)

\begin{tabular}{|c|c|c|}
\hline Conformação & Escore & Descrição \\
\hline \multirow{3}{*}{ Vista lateral } & 1 & $\begin{array}{l}\text { Articulação do tarso com ângulo maior que } 0 \\
\text { normal }\end{array}$ \\
\hline & 2 & Articulação do tarso com angulação intermediária \\
\hline & 3 & $\begin{array}{l}\text { Articulação do tarso com ângulo menor que } 0 \\
\text { normal }\end{array}$ \\
\hline \multirow{4}{*}{ Vista caudal } & 0 & Membros pélvicos com conformação paralela \\
\hline & 1 & $\begin{array}{l}\text { Membros pélvicos com articulação do tarso } \\
\text { levemente fechada }\end{array}$ \\
\hline & 2 & $\begin{array}{l}\text { Membros pélvicos com articulação do tarso } \\
\text { moderadamente fechada }\end{array}$ \\
\hline & 3 & $\begin{array}{l}\text { Membros pélvicos com articulação do tarso } \\
\text { intensamente fechada }\end{array}$ \\
\hline \multirow{4}{*}{ Locomoção } & 0 & Sem abdução dos membros \\
\hline & 1 & Leve abdução dos membros \\
\hline & 2 & Moderada abdução dos membros \\
\hline & 3 & Severa abdução dos membros \\
\hline
\end{tabular}




\section{Análises estatísticas}

A comparação do número de animais com alterações na conformação dos membros entre as diferentes categorias foi realizada pelo teste $\mathrm{X}^{2}$. Quando alguma das subcategorias apresentou um número de animais inferior a cinco, utilizou-se o teste exato de Fisher.

\section{Resultados e discussão}

Os resultados da avaliação de conformação dos membros pélvicos estão apresentados na Tabela 2 .

Os resultados de alterações na conformação dos membros pélvicos navistalateral não demonstraram alto número de animais acometidos, mas levando em consideração a faixa etária, foram identificados diferentes tipos de alterações para diferentes categorias. Para os animais com até uma lactação, a principal alteração que ocorreu foram animais com a articulação do tarso muito reta (escore 1). Para os animais com duas ou mais lactações, as alterações encontradas foram representadas por animais que apresentavam a articulação do tarso muito curva (escore 3). Esses achados foram semelhantes aos de Vermunt e Greenough (1996), que encontraram uma diminuição dos ângulos da articulação do tarso de acordo com o aumento da idade do animal. Os autores encontraram essa diminuição avaliando animais primeiramente aos 12 meses de idade e depois aos 24 meses de idade.

Tabela 2 - Distribuição dos escores de avaliação de conformação dos membros pélvicos na vista lateral, vista caudal e locomoção de fêmeas bovinas Holandesas de diferentes faixas etárias e número de lactações, de uma fazenda leiteira semi-intensiva localizada em Sete Lagoas, MG (julho de 2009)

\begin{tabular}{|c|c|c|c|c|c|c|c|c|c|c|c|c|c|c|}
\hline \multicolumn{15}{|c|}{ Conformação dos membros pélvicos } \\
\hline \multicolumn{5}{|c|}{ Vista lateral } & \multicolumn{4}{|c|}{ Vista caudal } & \multicolumn{6}{|c|}{ Locomoção } \\
\hline Categoria* & 1 & 2 & 3 & $1,3 \% * *$ & 0 & 1 & 2 & 3 & $1,2,3 \% *{ }^{* *}$ & 0 & 1 & 2 & 3 & $1,2,3 \%^{* *}$ \\
\hline C1 & 3 & 27 & 0 & $10,00^{A}$ & 23 & 7 & 0 & 0 & $23,33^{D}$ & 8 & 20 & 2 & 0 & $73,33^{c}$ \\
\hline $\mathrm{C} 2$ & 2 & 28 & 0 & $6,66^{A}$ & 15 & 9 & 6 & 0 & $50,00^{c}$ & 7 & 19 & 4 & 0 & $76,66^{\mathrm{BC}}$ \\
\hline C3 & 5 & 25 & 0 & $16,66^{A}$ & 12 & 10 & 7 & 1 & $60,00^{B C}$ & 9 & 15 & 6 & 0 & $70,00^{c}$ \\
\hline C4 & 1 & 29 & 0 & $3,33^{A}$ & 2 & 17 & 10 & 1 & $93,33^{A}$ & 1 & 16 & 12 & 1 & $97,67^{A}$ \\
\hline C5 & 4 & 26 & 0 & $13,33^{A}$ & 14 & 12 & 4 & 0 & $53,33^{c}$ & 6 & 19 & 2 & 3 & $80,00 \mathrm{BC}$ \\
\hline C6 & 0 & 25 & 5 & $16,66^{A}$ & 8 & 19 & 3 & 0 & $73,33 \mathrm{BC}$ & 11 & 12 & 7 & 0 & $63,33^{c}$ \\
\hline $\mathrm{C7}$ & 0 & 24 & 6 & $20,00^{A}$ & 6 & 16 & 7 & 1 & $80,00^{\mathrm{AB}}$ & 2 & 17 & 10 & 1 & $93,33 \mathrm{AB}$ \\
\hline
\end{tabular}

Nota: *As fêmeas bovinas $(n=210)$ foram divididas em sete categorias, cada qual com 30 animais. ${ }^{* *}$ Frequência de animais que apresentaram alterações nas avaliações de conformação dos membros pélvicos; as porcentagens foram calculadas em relação ao total de animais por categoria $(n=30)$. ${ }^{A-D}$ Médias seguidas por diferentes letras na mesma coluna diferem estatisticamente $(p<0,05)$ C1 - 0 a 3 meses; C2 - 3 a 6 meses; C3 - 6 a 12 meses; C4 - 12 meses até o parto; C5 - uma lactação; C6 - duas lactações; C7 - três ou mais lactações.

Boeeling e Pollott (1998), avaliando vacas Holandesas entre uma e quatro lactações, encontraram diminuição dos escores de conformação dos membros pélvicos na vista lateral. Os membros pélvicos se tornaram mais curvos da primeira até a terceira lactação. Na quarta lactação houve aumento do ângulo das articulações do tarso, sendo os resultados semelhantes aos animais de duas lactações. Já no presente experimento, os achados foram diferentes, uma vez que houve diminuição do ângulo das articulações do tarso nos animais das categorias C6 e C7.

Capion et al. (2008) encontraram alterações no ângulo das articulações do tarso em 33\% de novilhas 
aos 41 dias pré-parto, tendo esses valores diminuído após o parto para $26 \%$ nos animais com até 100 dias de lactação, 19\% nos animais entre 100 e 200 dias de lactação e $23 \%$ nos animais acima de 200 dias de lactação. 0 autor atribuiu essa diminuição ao descarte de animais, ao casqueamento e ao crescimento dos animais. No presente experimento, as novilhas foram as que menos apresentaram alterações dos membros pélvicos na vista lateral quando comparadas com todas as outras categorias $(3,33 \%$ dos animais com alterações), diferentemente dos achados de Capion et al. (2008). Estes, porém, avaliaram os mesmos animais antes do parto e na primeira lactação, enquanto neste trabalho foram avaliados diferentes animais em diferentes faixas etárias.

0 aumento dos escores da avaliação dos membros pélvicos na vista caudal ocorreu mais intensamente nos animais com idade superior a 1 ano, fase em que 93,33\% dos animais apresentaram alteração. Quando observados os animais na categoria com uma lactação (C5), entretanto, houve uma queda de quase metade das alterações Posteriormente, nas categorias C6 e C7, houve aumento dos índices de alterações dos membros pélvicos na vista caudal, porém sem atingir os valores dos animais da categoria $\mathrm{C} 4$.

Levando-se em consideração todos os animais acima de 1 ano de idade, verificou-se que dos 120 animais avaliados, 83,33\% apresentaram algum grau de alteração de fechamento da articulação do tarso. Esses valores foram semelhantes aos encontrados por Capion et al. (2008), que avaliaram os membros pélvicos na vista caudal de 122 novilhas Holandesas na Dinamarca e observaram que $81 \%$ tinham as articulações do tarso fechadas já antes do parto. Os autores também observaram que houve um pequeno aumento durante a lactação, em que $85 \%$ dos animais até os 100 dias de lactação e 86\% dos animais com mais de 100 dias de lactação apresentavam alterações.

Assim como na avaliação da conformação dos membros pélvicos na vista caudal, houve um aumento significativo dos escores de conformação do tipo locomoção nos animais com idade superior a 1 ano de idade. Dos animais avaliados entre 1 ano e o parto (C4), 97,67\% apresentaram abdução dos membros, com queda para $80 \%$ nos animais com uma lactação (C5) e queda ainda maior nos animais com duas lactações (C6). Nos animais com três ou mais (C7) lactações, esta conformação voltou a apresentar valores muito altos, acometendo $93,33 \%$ dos animais. Os animais entre 1 ano e o parto também apresentaram escores mais severos desta alteração, assim como a conformação dos membros pélvicos na vista caudal.

\section{Conclusão}

O grande número de alterações nas conformações dos membros pélvicos na vista caudal e locomoção mostram a importância de se realizar a seleção de animais com melhores aprumos para se prevenir e diminuir a ocorrência de conformações indesejáveis dos membros pélvicos. É importante também salientar que os animais entre 1 ano de idade e o parto (categoria C4) apresentaram números de alterações significativos, sendo necessários mais estudos para melhor avaliar essas alterações e se instituir medidas preventivas que evitem esses elevados valores de alterações.

\section{Referências}

Boelling D, Pollot GE. Locomotion, lameness, hoof and leg traits in cattle I: Phenotypic influences and relationships. Livest Prod Sci. 1998;54(3):193-203.

Boettcher PJ, Dekkers JCM, Warnick LD, Wells SJ. Genetic analysis of clinical lameness in dairy cattle. J Dairy Sci. 1998;81(4):1148-56.

Boettcher PJ, Jairath LK, Koots KR, Dekkers JCM. Effects of interactions between type and milk production on survival traits of Canadian Holsteins. J Dairy Sci. 1997;80(11):2984-95.

Capion N, Thamsborg SM, Enevoldsen C. Conformation of hind legs and lameness in Danish Holstein heifers. J Dairy Sci. 2008;91(5):2089-97.

Chapinal N, Koeck A, Sewalem A, Kelton DF, Mason S, Cramer G, et al. Genetic parameters for hoof lesions and their relationship with feet and leg traits in Canadian Holstein cows. J Dairy Sci. 2013;96(4):2596-2604. 
Dhakal K, Tiezzi F, Clay JS, Maltecca C. Short communication: Genomic selection for hoof lesions in first-parity US Holsteins. J Dairy Sci. 2015;98(5):3502-7.

Greenough PR, MacCallum FJ, Weaver AD. Lameness in cattle. Bristol : Wright Scientechnica; 1981. 471 p.

Laursen MV, Boelling D, Mark T. Genetic parameters for claw and leg health, foot and leg conformation, and locomotion in Danish Holsteins. J Dairy Sci. 2009;92(4):1770-7.

Logue DN, Offer JE, Kempson SA. Lameness in dairy cattle. Ir Vet J. 1993;46:47-58.

Olechnowicz J, Jaskowski JM, Nowak W. Effect of hind limb conformation on claw disorders in dairy primiparous cows. Bull Vet Inst Pulawy. 2010;54(3):437-9.

Pasman E, Reinhardt F. Genetic relationships between type composites and length of productive life of blackand-white Holstein cattle in Germany. Interbull Bull. 1999;(21):117-21.

Pérez-Cabal MA, Charfeddine N. Short communication: Association of foot and leg conformation and body weight with claw disorders in Spanish Holstein cows. J Dairy Sci. 2016;99(11):9104-8.
Politiek RD, Distl O, Fjeldaas T, Heeres J, McDaniel BT, Nielsen E, et al. Importance of claw quality in cattle: Review and recommendations to achieve genetic improvement. Livest Prod Sci. 1986;15(2):133-52.

Swalve HH, Alkhoder H, Pijl R. Estimates of breeding values for sires based on diagnoses recorded $t$ hoof trimming: Relationships with EBV for conformation traits. Interbull Bull. 2008;(38):87-90.

Vermunt JJ, Greenough PR. Hock angles of dairy heifers in two management systems. Br Vet J. 1996;152(2):237-42.

Warnick LD, Janssen D, Guard CL, Gröhn YT. The effect of lameness on milk production in dairy cows. J Dairy Sci. 2001;84(9):1988-97.

WHFF (World Holstein Friesian Foundation). Progress of type harmonization. 2008 [acesso 6 mar 2018]. Disponível em: https://tinyurl.com/y8nw5wym. 